\title{
Designing Focusing Solenoids for Superconducting RF Accelerators
}

\author{
G. Davis, V.V. Kashikhin, T. Page, I. Terechkine, J. Tompkins, T. Wokas
}

\begin{abstract}
The design of a focusing solenoid for use in a superconducting RF linac requires resolving a range of problems with conflicting requirements. Providing the required focusing strength contradicts the goal of minimizing the stray field on the surfaces of adjacent superconducting RF cavities. The requirement of a compact solenoid, able to fit into a gap between cavities, contradicts the need of mechanical support necessary to restrain electromagnetic forces that can result in coil motion and subsequent quenching. In this report we will attempt to address these and other issues arising during the development of focusing solenoids. Some relevant test data will also be presented.
\end{abstract}

Index Terms-Accelerator magnets, design methodology, magnetic analysis, protection, solenoids.

\section{INTRODUCTION}

For many years short solenoid lenses have been used as focusing elements in electron microscopes and other electron beam devices, like TV tubes, due to their ability to provide quality focusing. By using solenoids in transport channels, with some care, one can get quite modest emittance growth. Controlling the emittance growth is required to reduce particle losses in high power accelerators, where it is desirable to use smooth, axially symmetrical focusing with relatively short focusing period. This technique often works better than when pairs of quadrupoles are used that sequentially focus the beam in the horizontal and vertical plane, which results in significant halo formation [1]. Since the use of a solenoid as a focusing element is based on second order effects [2], it is desirable to go to higher magnetic field by employing superconducting devices to minimize the overall length occupied along the beamline. Such devices have been planned for the low energy part $(\sim 100 \mathrm{MeV})$ of a high power $\mathrm{H}^{-} \mathrm{RF}$ linac that is being studied at Fermi National Accelerator Laboratory [3]. The accelerating frequency in the linac is 325 $\mathrm{MHz}$ and the average current reaches $\sim 40 \mathrm{~mA}$. For this current, the required focusing period immediately after the RFQ section $(2.5 \mathrm{MeV})$ is $0.52 \mathrm{~m}$ with the integrated focusing strength of the lens

$$
F S=\int_{-\infty}^{\infty} B^{2}(z) d z \geq 1.8 T^{2} m \text {. }
$$

Manuscript received August 28, 2006.

Authors are with Fermi National Accelerator Laboratory (FNAL), P.O. Box 500, Batavia, IL 60510. Corresponding author I. Terechkine; phone: (630) 840-4017; e-mail: terechki@fnal.gov

Work supported by US DOE under contract No. DE-AC02-76CH03000
If the effective length of the focusing element is $0.1 \mathrm{~m}$, the average magnetic field in the device must be higher than 4.2 T. Inside a short solenoid, the magnetic field can not be considered uniform, so the maximum field can be much higher than the average. Since most of the accelerator uses superconducting accelerating structures, making focusing elements also superconducting and placing them inside the cryomodules provides an efficient arrangement. Employing superconducting accelerating structures imposes strict requirements on the strength of the magnetic field at the surfaces of all superconducting cavities. The level of this "fringe" field must be much lower than the earth's magnetic field $(\sim 50 \mu \mathrm{T})$ and according to some estimates should be on the level of $\sim 1 \mu \mathrm{T}[4]$.

\section{HISTORY}

The theory and practice of fabricating superconducting solenoids is far from being new (e.g. see [5] and [6]) and there are several vendors that could fabricate the needed focusing elements when provided with a proper set of requirements. The issue is that to develop the requirements took several cycles of iterative work on developing design concepts for the solenoids and accelerating cavities and attempts to integrate them into a cryomodule. Although there are several instances in the field of linear RF ion accelerators that target using focusing solenoids, adopting existing systems did not work well in our case. In the ISAC-II cryomodule (TRIUMF, Canada [7]), the focusing solenoids have $26 \mathrm{~mm}$ cold bore, $160 \mathrm{~mm}$ effective length, and $360 \mathrm{~mm}$ physical length. It uses two bucking coils wound above the main coil; the magnetic field in the bore reaches $9 \mathrm{~T}$. At SOREQ Research Center in Yavne, Israel, the accelerating module is under construction that also employs a superconducting solenoid as a focusing element [8] with a $35 \mathrm{~mm}$ cold bore, an $88 \mathrm{~mm}$ effective length, and a $280 \mathrm{~mm}$ physical length. Here, the magnetic field in the bore is $6 \mathrm{~T}$, and the solenoid uses active shielding wound above the main coil. The physical length of both devices mentioned above is more than could be allowed for our purpose. In addition, the magnetic field at the niobium surface of cavities for both systems is rather high, on the order of $0.01 \mathrm{~T}$, which makes it difficult to justify use of these or similar devices in our case. At MSU, an $80 \mathrm{MHz}$ accelerating system also employs a $9 \mathrm{~T}$ focusing solenoid fabricated by Cryomagnetics, Inc [9]. Here significant attention was devoted to shielding issues. First, reverse wound compensation coils were used to reduce stray magnetic field. Then, a niobium 
shield was installed around the solenoid to trap the magnetic field inside via the Meissner effect. Next, a low carbon steel vacuum vessel was used to reduce background magnetic field. Finally, a Cryoperm ${ }^{\circledR}$ shield was used around the cavities to reduce the magnetic field on the cavity walls to well below 1 $\mu \mathrm{T}$. This solenoid would meet the needs of our project, but it was too long $(\sim 300 \mathrm{~mm})$. So, it was necessary to find our own solution, which we approached in an iterative manner.

\section{Focusing Solenoid Design}

The following design algorithm was employed in the course of this study:

1. For each accelerating structure (defined by the type of cavities used), the initial specification for the focusing element was developed which included the required focusing strength, the level of fringe field, and the length of the lens;

2. For each version of a lens, a magnetic design solution was found;

3. To define physical length of each lens, a mechanical design of the cold mass and a cryostat was made;

4. To verify that the focusing elements and the cavities fit inside the allocated space, a layout of each accelerating section was made. The layout was also used to model beam dynamics;

5. Adjustments to the specifications were made and steps 1 to 5 were repeated as needed till the process converged;

6. Stress analysis of the cold mass was performed to ensure that at all stages of solenoid fabrication, cooling, and excitation, the stress in solenoid elements did not exceed allowable limits and that there was no separation of the coil from the bobbin;

7. Quench protection analysis was performed to find the maximum coil temperature and to define the protection strategy;

8. Prototype (or test) solenoids were designed, built, and tested to verify that the chosen design approach resulted in the expected performance of the system.

In the "front end" of the linac under study at FNAL, there are three distinctive sections [3]:

- A room temperature section employs accelerating cavities similar to drift tube linac structures, the socalled "cross-bar H-type" $(\mathrm{CH}) \mathrm{RF}$ structure. This section accelerates particles up to $\sim 10 \mathrm{MeV}$ and uses superconducting solenoids in individual cryostats for focusing;

- Two superconducting single-spoke cavity sections (SS-1 and SS-2) accelerate particles from $10 \mathrm{MeV}$ to $\sim 30 \mathrm{MeV}$ and then from $30 \mathrm{MeV}$ to $\sim 100 \mathrm{MeV}$. Two versions of focusing solenoids are used in these sections. A common cryostat is used to house the cavities and the solenoids in each of these sections.

Table I summarizes the requirements for the focusing solenoids in the room temperature section and in the superconducting sections resulting from several iterations of the design cycle.

TABLE I

MAJOR REQUIREMENTS FOR FOCUSING SOLENOIDS

\begin{tabular}{llll}
\hline \hline & \multicolumn{3}{c}{ Section Type: } \\
Parameter: & $\mathrm{CH}$ & SS-1 & SS-2 \\
\hline Bore diameter (mm) & 20 & 30 & 30 \\
Bore type & warm & cold & cold \\
Integrated Strength (T2·m) & 1.8 & 3 & 5 \\
Field margin (\%) & 30 & 30 & 30 \\
Fringe field @ cavity surface (T) & $<10^{-4}$ & $\sim 10^{-6}$ & $\sim 10^{-6}$ \\
Cold mass length (mm) & 130 & 220 & 295 \\
Cryostat type & Individual & Common & Common \\
\hline \hline
\end{tabular}

The only difference between the solenoids for the SS- 1 and SS-2 sections is in their focusing strength. The required integrated strength grows with energy; if the energy is higher than $\sim 100 \mathrm{MeV}$, using solenoids for focusing becomes less practical than using quadrupole lenses. As the energy increases, the particle beam becomes more "rigid" and switching to quadrupoles for focusing does not result in noticeable degradation of the beam quality.

\section{MAgnetic Design}

The main goal of this stage of the design activity was to find a configuration of a solenoid that would meet the major requirements. This appeared to be more difficult for the $\mathrm{CH}$ section because of the relatively small available space between the adjacent low- $\beta$ accelerating cavities. The need for a separate cryostat, cavity outer tank, connection flanges and bellows contributed to the problem. Although the cavities are at room temperature here, to prevent multipacting, it is not desirable to have a significant magnetic field in the area with the RF accelerating field. To reduce the fringe magnetic field, bucking coils on both sides of the main coil of the solenoid are used, which makes the real estate problem even worse.

To obtain reliable performance of the focusing element in the machine, a proper reserve on the solenoid integrated strength must be considered. At the design stage, before reliable test data are available, $30 \%$ of the current margin is used. Taking this high margin also results in increased length of the system; as prototype solenoids are fabricated and tested, this margin can be set to a lower level.

The obvious idea of using $\mathrm{Nb}_{3} \mathrm{Sn}$ strand to get higher field was rejected because this led us to significant complications of the coil fabrication process and a corresponding cost increase. A graded coil design seemed attractive, but after a thorough analysis it was determined that the design goal could be reached if some care was exercised to obtain a high packing factor in the multilayer regular winding. Studies of several mockup coils, wound using round and rectangular strands and different types of ground and interlayer insulation, led to this conclusion. It was found possible to rely on a packing factor as high as 0.75 for the main coil and 0.68 for the bucking coils. The main design features of the $\mathrm{CH}$ section solenoid are shown in Fig. 1. The solenoid includes the main coil, two 
bucking coils, and the flux return with side flanges. The main coil collar helps prevent separation of the coil's inner layer from the bobbin, which could happen during excitation. The flux return flanges catch magnetic field that was not deflected by the bucking coils. Although the flux return gets quite saturated at the maximum current, it reduces the fringe field to the desired level. The main parameters of the solenoid can also be found in Fig. 1 [10].

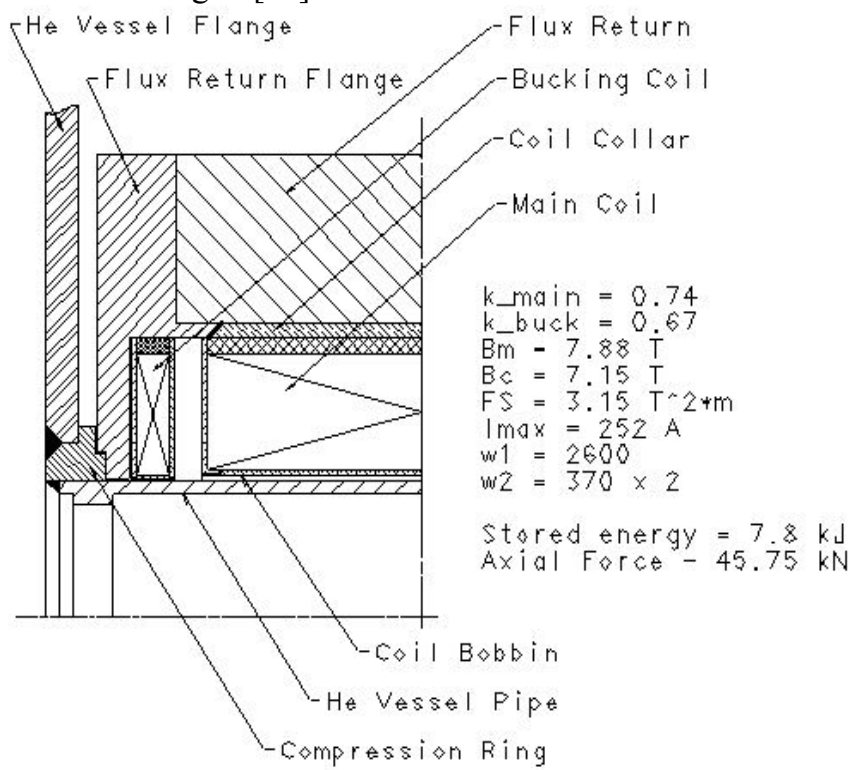

Fig. 1. $\mathrm{CH}$ section solenoid: configuration and main parameters.

Due to the decision of use a separate warm bore cryostat in the $\mathrm{CH}$ section, the inner diameter of the coil could not be made smaller than $55 \mathrm{~mm}$. The length of the cold mass is 130 $\mathrm{mm}$ (which does not include the He vessel flange in this case) and the length of the main solenoid is $\sim 96 \mathrm{~mm}$. The outer diameter of the flux return is $\sim 175 \mathrm{~mm}$.

The maximum field and current in the solenoid are found by using the known technique of locating the crossing point of the solenoid 'load line' with the superconductor critical surface. In the case of the $\mathrm{CH}$ section solenoid at $4.2 \mathrm{~K}$, the maximum field in the coil is $7.88 \mathrm{~T}$ at a current of $\mathrm{I}=252 \mathrm{~A}$ and. This high field requires the mechanical design to prevent separation of the inner layer of the coil from the bobbin, which could result in quenching.

\section{Mechanical Analysis}

The stress and deformation in the solenoid is different for different stages of fabrication and also depends on the environment temperature. While a coil is being wound, some strand tensioning is usually used. The tension transforms into tangential stress and is responsible for a change in the normal stress in the body of the coil at equilibrium. As a result, during winding, a significant stress accumulation can occur in the bobbin.

During cool down, due to the contraction of different materials used in the coil, stress in the bobbin can exceed the allowable limit. When the coil is energized, the magnetic volumetric force acts to relax the stress in the bobbin. The action of this force can result in separation of the inner layer of the coil from the bobbin. This situation is potentially dangerous (especially if epoxy impregnation is used, as in this design): the movement and associated friction can generate sufficient heat to quench the solenoid.

By properly choosing the strand tension force during winding, overlap between the collar and the coil (see Fig. 1), and the material properties of the collar and the bobbin, it was possible to have the coil remaining in contact with the bobbin during excitation with the stress not exceeding allowable limit in any part of the solenoid (see [11]-[13]).

As bucking coils work to deflect the total flux of the main coil into the flux return, a significant repulsing axial force of $\sim 45 \mathrm{kN}$ is generated. It was shown in [10] that this force can not be compensated by the flux return flange, made of low carbon steel and annealed to improve its magnetic properties. To provide additional support, a compression ring is introduced (Fig. 1) that is used as follows:

1. A compressive force of $\sim 15 \mathrm{kN}$ is applied to the solenoid assembly through the compression ring. This eliminates all gaps between parts of the assembly;

2. A tensioning force of $\sim 15 \mathrm{kN}$ is applied to the $\mathrm{He}$ vessel inner pipe by using a special fixture;

3. The compression ring is welded to the inner pipe while it is under tension;

4. The assembly fixture is removed, leaving the coil under compression through the compression ring and due to the stretched pipe.

Analysis of this procedure for the CH-type solenoid was performed in [14], taking into account additional effects induced by contraction during cooling down to LHe temperature. The analysis revealed some problems with the initial design and suggested ways to improve it.

Once a stable mechanical configuration is obtained, the quench behavior of the solenoid can be evaluated.

\section{QUENCH BEHAVIOR}

For a variety of reasons [6], [7], the local temperature in the coil winding can exceed the critical temperature of superconducting strand, resulting in the propagation of a normal zone. Depending on the low-temperature properties of materials used in the solenoid and on the details of coil design, this process potentially can lead to irreversible damage of the coil. Knowing the details of quench behavior is important in developing a quench protection system. This system must detect the appearance of a quench, shut down the external power source, and let the solenoid discharge through an optional external resistor to dissipate its stored energy outside the cryostat.

A simple method has been developed that allowed analyzing propagation of the normal zone in the coil [15]. This method was used to predict quench behavior of "test" coils that were built to check on the main coil mechanical, thermal, and quench behavior [16]. The method provided the temperature distribution in the test coils at any time after the initial transition, voltage to ground time profiles for any layer, and finally, a current decay curve with or without an external 
dump resistor. Comparing the results of mechanical and thermal analysis with the data obtained during the testing of the test coils [17], we were able to evaluate the reliability of our modeling. Fig. 2 shows comparison of the predicted current decay (in the case when no external dump resistor is used) with the measured one.

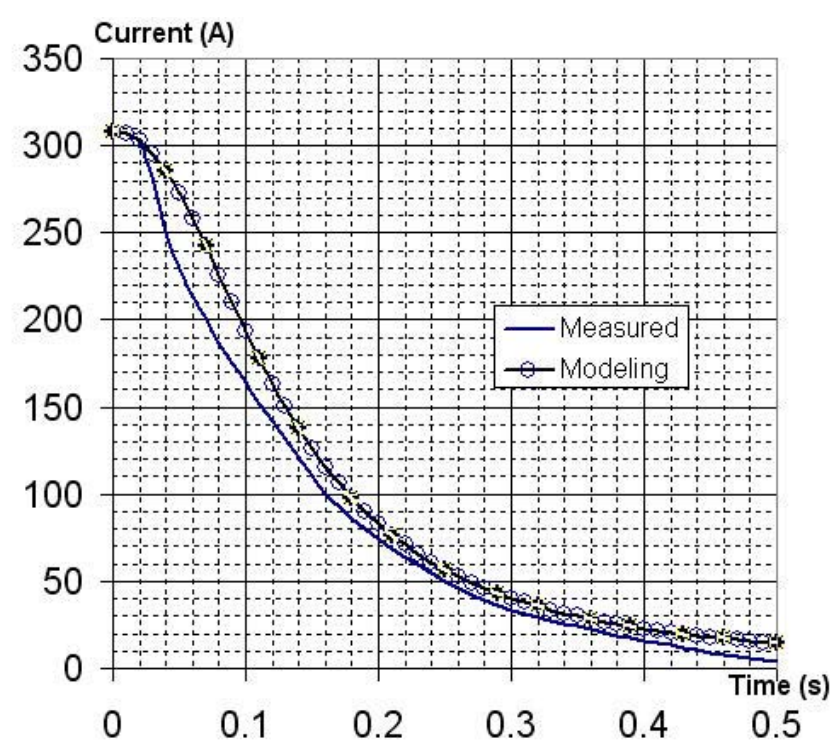

Fig. 2. Shorted solenoid current decay after quenching at maximal current

The measured current decay immediately after the quench initiation is faster than the predicted one, probably because of the presence of some resistance in the circuit (the copper power leads and protecting diodes). Because the current shape reflects the change of the coil resistance with time, the good agreement is an indication that the modeling provides a quite adequate picture of the normal zone propagation and of the coil temperature. The same conclusion was reached after analyzing the test coil voltage tap signals [18].

\section{SHIELDING}

The main difference between the SS section solenoids and the $\mathrm{CH}$ section solenoids is that the SS sections, employing superconducting cavities, require a very small fringe field. The smaller inner diameter of the solenoids in the SS section does not significantly reduce the fringe field relative to the $\mathrm{CH}$ solenoid design, so additional mu-metal shielding is required. To properly design this shielding, it is necessary to know how the fringe field changes in respond to uncertainties during coil fabrication, including variations in coil dimensions, packing factor, and material properties.

A thorough analysis of the problem was performed in [19] which shows that the presence of the low carbon flux return effectively reduces all fluctuations of the fringe field due to all uncertainties to the level of $\sim 10^{-5} \mathrm{~T}$. This opens the way to design the shielding, made of Cryoperm ${ }^{\circledR}$, for installation in the cryostat.

The main parameters of the design are the number of layers in the shielding and their thickness. All openings in the shielding for power and instrumentation leads must be protected by using cylindrical wells of appropriate depth. Reaching the required level of fringe field on the walls of the RF cavities $\left(10^{-6} \mathrm{~T}\right)$ seems to be an achievable, although difficult, task.

\section{CONCLUSION}

Following the algorithm described above, the designs of the $\mathrm{CH}$ section and the SS-1 section solenoids have been made. The $\mathrm{CH}$ section solenoid has been fabricated and is to be tested in September 2006. Results of the testing and associated magnetic measurements will be used to finalize the design of the SS-1 solenoid. Testing the first SS-1 prototype is planned for the beginning of 2007.

\section{REFERENCES}

[1] A. N. Mirzoian, P. N. Ostroumov, G. V. Romanov, and A. P. Fateev, "Increase of injection efficiency in a high current linear proton accelerator," Journal of Technical Physics, v. 25, pp. 710-713, 1980.

[2] M. Reiser, Theory and design of charged particle beams, John Wiley and Sons, Inc., N. Y., 1994.

[3] P. N. Ostroumov, K. W. Shepard, G. W. Foster, I. V. Gonin, and G. V. Romanov, "Front end design of a multi-GeV H-minus linac," PAC-05, Proceedings, pp. 3286-3288, 2006.

[4] Handbook of accelerator physics and engineering, ed. A. W. Chao and M. Tigner, World Scientific Publishing Co.. Pte. Ltd., 1998, p. 531.

[5] D. Bruce Montgomery, Solenoid magnet design, John Wiley and Sons, Inc., N. Y., 1969.

[6] Martin N. Wilson, Superconducting magnets, Oxford University Press, Oxford, 1983.

[7] R.E. Laxdal, "Superconducting RF developments and prototype commissioning at IASC II," 34-th ICFA Advanced Beam Dynamics Workshop on High Power Superconducting Ion, Proton, and MultiSpecies Linacs, May 22-24, 2005, Naperville, Il, USA, http://www.niu.edu/clasep/HPSLconf/proceedings.html

[8] M. Pekeler, K.Dunkel, C. Piel, H. Vogel, P. vom Stein, and N. Pupeter, "Development of a superconducting RF module for acceleration of protons and deuterons at very low energy," 34-th ICFA Advanced beam Dynamics Workshop on High Power Superconducting Ion, Proton, and Multi-Species Linacs, May 22-24, 2005, Naperville, Il, USA, http://www.niu.edu/clasep/HPSLconf/pdf/59.pdf.

[9] M. Johnson, et al., "Cryomodule design for a superconducting linac with quarter-wave, half-wave, and focusing elements," PAC-05, Proc., pp. 4317-4319, 2006.

[10] G. Davis, V. V. Kashikhin, T. Page, I. Terechkine, and T. Wokas, "Linac CH-type cavity section focusing solenoid cold mass design," TD06-020, FNAL, March 2006.

[11] I. Terechkine, "Analysis of stress in PD front end solenoids," TD-05039, FNAL, Sept. 2005.

[12] I. Terechkine, "Test solenoid design proposal," TD-05-040, FNAL, Sept. 2005.

[13] B. Wands, "A finite element simulation of the winding, cooldown, and energizing of the test solenoid," TD-06-019, FNAL, March 2006.

[14] B. Wands, "Summary of DTL section solenoid FEA," TD-06-043, FNAL, Aug. 2006.

[15] P. Bauer, I. Terechkine, "Proton driver front end focusing solenoid quench protection studies. Part 1: Method description and the first iteration," TD-06-003, FNAL, January, 2006.

[16] I. Terechkine, "Proton driver front end focusing solenoid quench protection studies. Part 2: Test solenoid quench protection," TD-06-004, FNAL, January, 2006.

[17] R. Carcagno, et al., "Superconducting solenoid model magnet test results," ASC-06, report 5LG01, to be published.

[18] R. Carcagno, et al., "Test solenoids: expected performance and test results," TD-06-027, TD-06-028, and TD-06-029, FNAL, July 2006.

[19] G. Davis, V. V. Kashikhin, T. Page, I. Terechkine, and T. Wokas, "Linac SS-I section focusing solenoid prototype cold mass design, TD06-041, FNAL, January 2006. 